\title{
Effect of Determining Initial Conditions by Four-Dimensional Variational Data Assimilation on Storm Surge Forecasting
}

\author{
S.-Q. Peng and L. Xie* \\ North Carolina State University, Raleigh, NC
}

\section{Introduction}

In the past half century, tremendous progress has been made in numerical prediction of storm surge (Jelesnianski et al., 1992; Xie et al., 2004; Peng et al., 2005) and coastal ocean circulation (Blumberg and Mellor, 1987). Although improvements have been made, substantial prediction errors still exist (Houston et al., 1999). Numerical ocean predictions are never exact solutions of the real world ocean. Instead they are only an approximation of the real ocean both in terms of dynamics and physics. The errors or uncertainties of model prediction come from two main sources: 1) dynamical simplifications and physical parameterizations; and 2) initial and boundary conditions. Therefore, oceanic prediction can be improved either by improving the dynamical approximations and physical parameterizations or by improving initial and boundary conditions. Significant improvements have already been made in modeling the ocean dynamics and physics. With the development of large ocean observing systems and remote sensing techniques, more and more oceanic data are becoming available. This provides a promising prospect for improving the model initial conditions through data assimilation. As a result, data assimilation has become widely used in meteorological and oceanographic predictions in recent years.

Among all data assimilation methods, 4dimensional variational data assimilation (4D-Var) is one of the most effective and powerful approaches developed over the past two decades (Le Dimet and Talagrand 1986; Courtier et al. 1994). It is an advanced data assimilation method which involves the adjoint technique and has the advantage of directly assimilating various observations distributed in time and space into the numerical model while maintaining dynamical and physical consistency with the model. Thus, 4D-Var has been widely applied in meteorological and oceanographic data assimilation, sensitivity studies, and parameter estimation.

While the adjoint approach has been applied in global ocean analyses and prediction using real observations and realistic Ocean General Circulation Models (OGCM) (e.g., Martel and Wunsch 1993; Oldenborgh et al. 1999; Bonekamp et al. 2001; Weaver et al. 2003; Köhl and Stammer 2004), the application of the adjoint approach in the coastal short-range ocean forecasting is still more theoretical than practical using very simple ocean models or psuedo-observation data. It is worth noting some pioneering studies in this area, such as those of Bennett and Mclntosh (1982), Yu and O'Brien (1991, 1992), Das and Lardner (1991), Seiler (1993), Lu and Hsieh (1997, 1998a,b), Heemink et al. (2002), and Zhang et al. (2002 and 2003). The results of all of these studies have indicated that the adjoint variational approach is quite effective and robust in regional ocean data assimilation, sensitivity studies and parameter estimation and may benefit real-time prediction of oceanic circulation and sea state. The studies mentioned above, however, mainly focused on investigating the feasibility of the adjoint method in ocean research and forecasting under simplified dynamics and numerics. The models used in these studies were simplified either physically (e.g., quasigeostrophic approximation, shallow water approximation, or reduced gravity approximation) or numerically (e.g., one-dimensional or twodimensional assumptions) due to computational considerations. The questions are:

1) With the advent of more powerful supercomputing capabilities, is it practical to develop a 4D-Var algorithm for three-dimensional primitive equation coastal ocean models now or must we still resort to physical or numerical simplification to reduce computing cost?

2) What is the best strategy for data assimilation, as more and more coastal observing systems are deployed, various observations from moorings, buoys, ships, coastal radars and satellites become available in near real-time? Should we assimilate all available data simultaneously or identify a small subset of data sets which are most effective?

3) Storm surge forecasting often relies on a cold start of the numerical model (i.e., without initialization). Is this an acceptable practice? How important or effective is initialization in storm surge forecasting?

To answer these questions, an adjoint model of the three-dimensional, nonlinear primitive equation, Princeton Ocean Model (POM) (Mellor 2003; Blumberg and Mellor 1987) is developed. A 4D-Var algorithm based on the POM and its adjoint model is configured as the first step of our goal of performing real-time data assimilation and operational oceanic nowcasting/forecasting using the adjoint variational approach. Experiments with pseudo-observations generated by a different version of POM with higher

\footnotetext{
* Address of correspondence: Prof. Lian Xie, NCSU/MEAS, Box 8208, Raleigh, NC 27696-8208, U.S.A.

Email: xie@ncsu.edu.
} 
resolution are conducted to verify and evaluate the developed 4D-Var algorithm.

\section{The nonlinear POM and its tangent linear and adjoint model development}

The Princeton Ocean Model (POM) 2002 version (referred to as pom2k) is used for the forward prediction model in this study. The POM is a threedimensional, primitive equation ocean model which includes a simplified version of the level 2.5 Mellor and Yamada turbulent closure scheme (Mellor and Yamada, 1982, hereafter denoted as MY82, details can be found in Mellor, 1989). In this study, radiation open boundary conditions are applied to seaward boundaries. The bottom terrain-following, sigma coordinate equations of the POM as well as a full description of the POM can be found in Blumberg and Mellor (1987) and Mellor (2003). The governing equations of the POM can be written in a general form as,

$$
\begin{gathered}
\frac{\partial \mathbf{x}}{\partial t}=F(\mathbf{x}) \\
\left.\mathbf{x}\right|_{t_{0}}=\mathbf{x}_{0} \\
\left.\mathbf{x}(t)\right|_{\Gamma}=\mathbf{y}(t)
\end{gathered}
$$

where $\mathbf{x}$ represents the vectors of model state variables, which includes current velocity $U$ and $V$, temperature $\mathrm{T}$, salinity $\mathrm{S}$, surface elevation $\eta$, and turbulence quantities $q^{2}$ and $q^{2} l . \mathbf{x}_{0}$ and $\mathbf{y}(t)$ represent the initial condition (IC) at initial time $t_{0}$ and lateral boundary condition on $\Gamma$, respectively.

The TLM of the POM can be obtained by linearizing the POM forecast model (1) about a nonlinear model trajectory $\mathbf{x}(t)$ and $\mathbf{y}$ :

$$
\begin{gathered}
\frac{\partial \mathbf{x}^{\prime}}{\partial t}=\frac{\partial F(\mathbf{x})}{\partial \mathbf{x}} \mathbf{x}^{\prime} \\
\left.\mathbf{x}^{\prime}\right|_{t_{0}}=\mathbf{x}_{0}^{\prime} \\
\left.\mathbf{x}^{\prime}(t)\right|_{\Gamma}=\mathbf{y}^{\prime}(t)
\end{gathered}
$$

where prime represents perturbations of the corresponding variables. The adjoint model corresponding to (2) is

$$
\begin{aligned}
-\frac{\partial \widehat{\mathbf{x}}}{\partial t} & =\left(\frac{\partial F(\mathbf{x})}{\partial \mathbf{x}}\right)^{T} \widehat{\mathbf{x}} \\
\left.\widehat{\mathbf{x}}\right|_{t_{R}} & =0 \\
\left.\widehat{\mathbf{x}}(t)\right|_{\Gamma} & =0
\end{aligned}
$$

where $\hat{\mathbf{x}}$ represents the vector of adjoint variables and $t_{R}$ the ending time of forward integration. The negative sign in the left-hand-side of (3a) indicates that the adjoint model integrates backward in time.

Considering the complication of the time-splitting scheme and the high nonlinear feature of turbulence closure in POM, the TLM and adjoint model of POM are developed directly from the POM forward model code by hand-coding. As pointed out by the ROMS TLM/adjoint model developing group (Moore et al, 2004), hand-coding allows a better understanding and control of the code structure of the resulting models.

\section{Experiment design}

To verify and evaluate the performance of the 4D-Var algorithm based on the POM, we apply the algorithm to a storm surge case along the United States East Coast during hurricane Hugo, Sept. 2122,1989 . The "pseudo-observations" generated by a high resolution model which is described in detail in Xie et al. (2004) and Peng et al. (2004, 2005) are used. Using "pseudo-observations" in data assimilation studies has the advantage of providing a full suite of balanced data sets which can be assimilated into the forecast model (Zhang et al., 2002, 2003). In this study, the same wind field is used to generate the "pseudo-observations" and for the storm surge forecast, so the uncertainty associated with the wind forcing is minimized. This allows us to focus on the effect of determining initial conditions on storm surge.

The numerical experiments are designed as:

NoDA: 9-h model run with original IC from a 12-h spin-up (control run);

DA-1: 9-h model run with optimal IC from 4D-Var of water level only;

DA-2: 9-h model run with optimal IC from 4D-Var of both water level and surface currents;

The horizontal resolution for the four experiments is 2' $x 2$ ' (about $3.1 \times 3.7 \mathrm{~km}$ ) with a total number of grid points of $136 \times 106$ and 3 vertical sigma levels. The time step is 3 minutes. Inflow boundary conditions are used for water level, radiation boundary condition for 3-D currents, and upstream advection boundary conditions for temperature, salinity and turbulent kinetic energy. In order to generate a large error in the control run so that improvements by 4D-Var can be seen clearly, a fixed boundary condition is applied to the vertically-averaged currents. The wind stress are calculated by using the Holland hurricane wind model (Holland, 1980) and updated every 10 minutes. The control run and the 4D-Var experiments started at $21 Z$ Sept. 21. A 12-h spin-up is run and the output from the spin-up is used as the initial condition of the NoDA experiment as well as the first guess field for the 4D-Var experiments (DA-1 and DA-2 ).

The control variables in the 4D-Var experiments are water level, current (including 3-D current and 2D vertically integrated current) field, temperature, salinity, and bottom stress. The pseudo-observations of water level and surface currents on each ocean grid point are generated by a different version of the POM running at double horizontal resolution (or half grid size) and with an inundation/drying scheme (Xie et al. 2004) which enables the coastal boundary to be time-dependent. A 3-h data assimilation window from $21 Z$ Sept. 21 to $00 Z$ Sept. 22 is set for the 4D- 
Var experiments with a 10-minute sampling frequency. The cost function is defined as:

$$
J\left(\mathbf{x}_{0}\right)=\left(\mathbf{x}_{0}-\mathbf{x}_{b}\right)^{T} \mathbf{B}^{-1}\left(\mathbf{x}_{0}-\mathbf{x}_{b}\right)+\alpha \sum_{r}^{M} \sum_{i}^{N}\left(\mathbf{P}_{r, i}-\mathbf{P}_{r, i}^{o b s}\right)^{T} \mathbf{R}^{-1}\left(\mathbf{P}_{r, i}-\mathbf{P}_{r, i}^{o b s}\right)
$$

where $\mathbf{x}_{0}$ represents the vector of control variables at the initial time $t_{0}$, and $\mathbf{x}_{b}$ represents their background values. $\quad \mathbf{P}_{r, i}$ and $\mathbf{P}_{r, i}^{\text {obs }}$ denote the model simulated values and the observations of water level or surface currents, respectively, at location $i$ and time level $r . \mathrm{N}$ is the number of grid points over the ocean and $M$ the number of time levels of observations. $\mathbf{B}$ and $\mathbf{R}$ are the error covariance for the background vector and the observations, respectively. $\alpha$ is a weighting parameter which makes the magnitudes of each observation term of (4) in balance.

\section{Results}

Fig. 1 shows the evolution of the total cost function and each of its terms with respect to the number of iterations for Experiment DA-1. The evolution of the total cost function is similar to that of the observation term, which decreases rapidly during the first 10 iterations and then varies slowly. The values of the background term, which measures the difference between the background field and the initial condition at each iteration, is zero at the $0^{\text {th }}$ iteration and increases with the number of iterations as the initial conditions are adjusted to fit the model trajectory to the "observations". The evolution of the cost function with respect to the number of iterations for DA-2 is similar to that for DA-1.

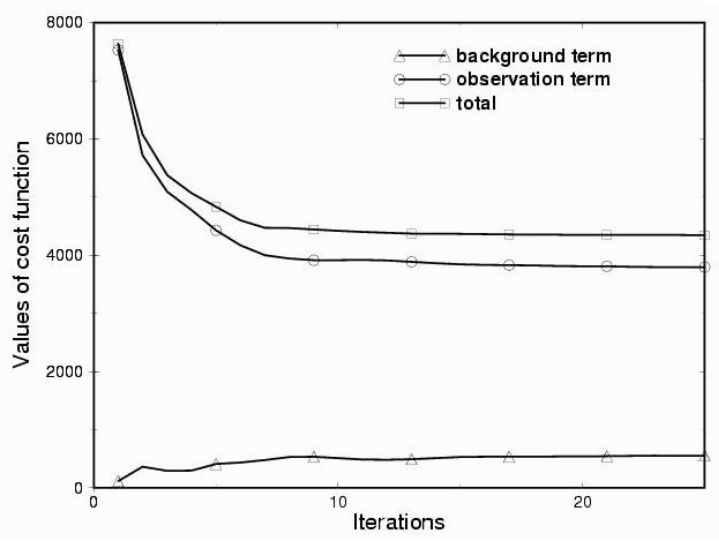

Fig. 1 The evolution of each term of the cost function with respect to the number of iterations for DA-1.

Fig. 2 shows the water level fields from the "observations" and each experiment at 01z Sept. 22 which is out of the assimilation window. Compared to the "observations" (Fig. 2a), the control run without data assimilation (NoDA, Fig. 2b) under-predicts the water level along much of the coastline north of the Georgia-
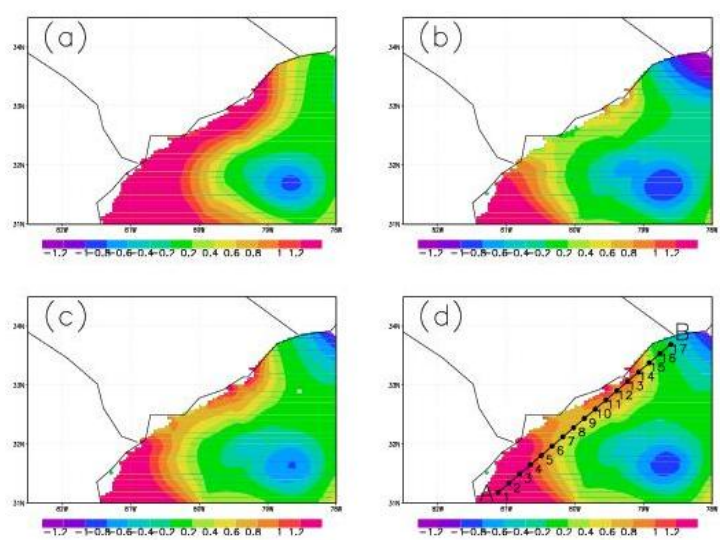

Fig. 2 The water level fields at $00 Z$ Sept. 22 from (a) pseudo-observations; (b) NoDA; (c) DA-1 and (d) DA-2 (line $A B$ and the numbers along it are for figs 4-5) (unit: $m$ ).

South Carolina border $\left(32^{\circ} \mathrm{N}\right)$. After assimilating the water level (Fig. 2c), the height of the water level over this area increases and is closer to the "observations". Assimilating both water level and surface currents (Fig. 2d) has similar results as assimilating only water level. Fig. 3 shows the time series (starting at $21 Z$ Sept. 21) of the root mean square error (RMSE) of water level averaged over all ocean grid points for each experiment with respect to the "observations" of water level. The model forecasting errors are reduced significantly by data assimilation within and a few hours beyond the assimilation window, with DA-2 slightly outperforming DA-1. However, the effect of data assimilation outside the assimilation window decreases as forecast time increases.

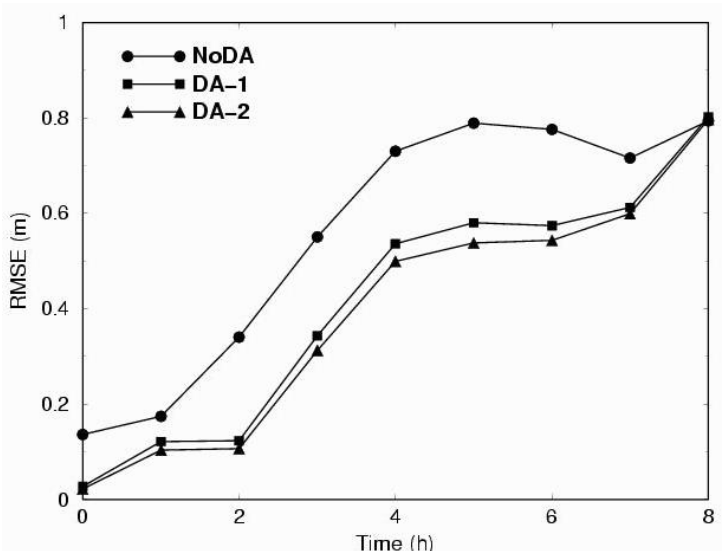

Fig. 3 Time series of the root mean square error (RMSE) of water level averaged over all ocean grid points for each experiment with respect to the pseudo-observations of water level starting from $21 Z$ Sept. 21 to $05 Z$ Sept. 22 (unit: $\mathrm{m})$.

The height of the peak storm surge along the coast is often the quantity of interest during the threat of a tropical cyclone. Fig. 4 shows the peak surge at 17 locations evenly distributed along a line parallel to 


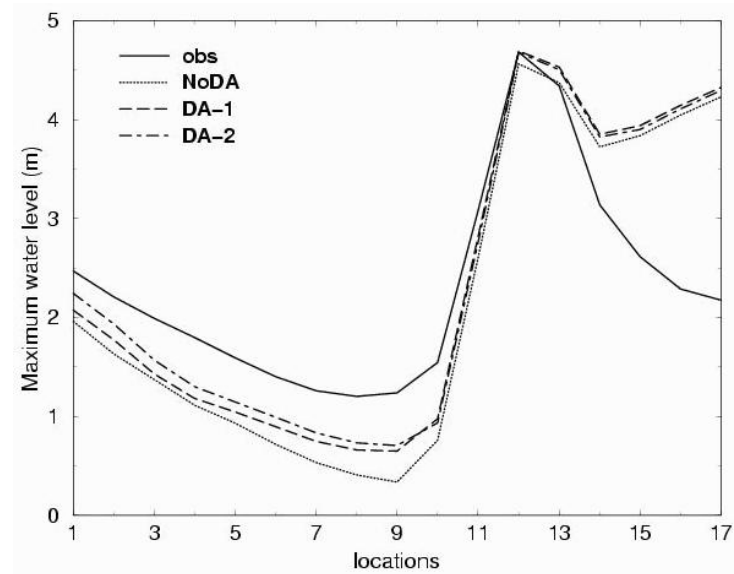

Fig. 4 Maximum height of water level along line $A B$ shown in Fig. $2 \mathrm{~d}$ for the pseudo-observations and each experiment (the number 1 in $\mathrm{X}$-axis corresponding to location $\mathrm{A}$ and 17 corresponding to $B$. unit: $m$ ).

the coast (line AB in Fig. 2d) for the "observations" and each experiment. It indicates that data assimilation produces significant improvements in the estimation of peak surge along the southern section of line $A B$, but no improvement on the northern section of the line. It is worth noting that although the storm surge predicted by the stand-alone POM without data assimilation produced large errors north of $32^{\circ} \mathrm{N}$ as shown in Fig. 2, it is able to capture the peak surge that occurred near location 12. As a result, the improvement in peak surge is small near location 12 . The large error in peak surge that occurred near the northern boundary (locations 15-17) is not effectively reduced by data assimilation. The error in this region is apparently less sensitive to initial conditions. This could be the result of model deficiencies, such as the lower resolution used, the lack of an inundation/drying scheme, and the fixed lateral open boundary conditions for the verticallyaveraged 2-D current. The time series of water level of 4 points $(7-10)$ located in the middle section of the line
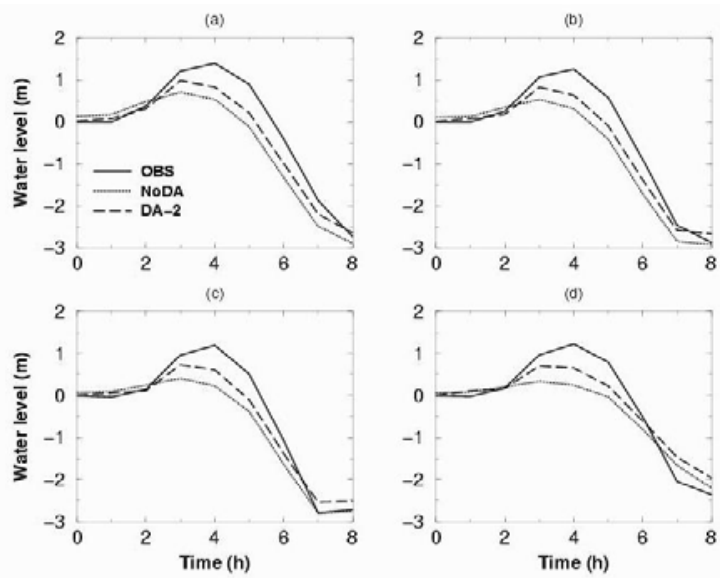

Fig. 5 The evolution of water level over 4 selected points (6-9) located on the middle section of line AB (Fig. 2d) for pseudoobservations, NoDA and DA-2, starting at 00z Sept. 22 (unit: $\mathrm{m})$.
AB for "observations", NoDA and DA-2 are shown in Fig. 5a-d. The figures show that data assimilation leads to significant improvements in storm surge prediction during the simulation period.

The surface current fields (figures omitted) show that assimilating only water level or assimilating both water level and surface currents intensifies the vortex and produced onshore currents which leads to an increase in the water level along the coast. It is interesting to note that, although assimilating both water level and surface currents have larger impacts on the model simulation of surface currents, assimilating water level alone can produce a comparable improvement in storm surge forecasting. Because the 4D-Var approach adjusts all control model variables simultaneously through the model dynamics and physics when assimilating one or more types of observations, assimilating water level leads to significant impacts on the surface currents.

\section{Summary and discussion}

In this study, the tangent linear model and the adjoint model of the 3-D primitive equation coastal ocean circulation model (POM) are developed. Experiments for a storm surge case are conducted on a single Linux-based $2.8 \mathrm{GHz}-3.2 \mathrm{GHz}$ Xeon processor to evaluate the potential application of a 4D-Var algorithm based on the POM adjoint model in storm surge forecasting by assimilating pseudoobservations of water level and surface currents into the model. For a 12-hour forecast with a 3-h data assimilation window (with 10 iterations of minimization) with the setup used in this study, it requires approximately half an hour of CPU time. Thus, real-time ocean forecasting using a threedimensional primitive equation model with 4D-Var data assimilation is not beyond reach. When a multiprocessor parallel computer cluster is used, higher resolutions can be achieved to meet the requirements of today's operational ocean forecasting needs.

The experimental results demonstrate that the 4D-Var data assimilation based on the developed POM adjoint model is able to find an "optimal" initial condition for the storm surge forecasting, with the values of the cost function which measures the difference between the model and "observations" reducing rapidly during the first 10 minimization iterations. Improvements on water level prediction are obtained both within and several hours beyond the assimilation window by assimilating water level "observations" alone or assimilating both water level and surface current "observations". The added benefit of assimilating both water level and surface currents is relatively small since water level and current fields are adjusted in dynamical and physical consistency with the constraint of the model control equations and the cost function.

For the storm surge case studied in this paper, notable improvements are obtained by finding an "optimal" initial condition through data assimilation. However, the improvements decrease rapidly in time 
beyond the assimilation window. The effect of data assimilation only lasts for several hours beyond the data assimilation window. Therefore, only changing the initial condition through data assimilation does not ensure an accurate forecast of storm surge with a long lead time.

Note that the same Holland wind model is used to generate the hurricane winds for the prediction model as well as the model which generates the "pseudoobservations". Thus, the forecast errors are the result of the differences between the two models. The datageneration model is run at higher resolution and also includes an inundation scheme, whereas the forecast model is configured at a lower resolution and does not couple to an inundation model. Thus, the results presented above show that forecast errors due to deficiencies in model physics or numerics can not always be effectively corrected through improving initial conditions alone.

Acknowledgement: This study is supported by the Carolina Coastal Ocean Observation and Prediction System (Caro-Coops) project under NOAA Grant No. NA16RP2543 and the Coastal Ocean Research and Monitoring Program (CORMP) under NOAA Grand \#NA16RP2675 via the National Ocean Service, through Charleston Coastal Services Center. CaroCOOPS is a partnership between the University of South Carolina and North Carolina State University, and CORMP is a partnership between the University of North Carolina at Wilmington and North Carolina State University.

\section{References}

Bennett, A. F., and P. C. Mclntosh, 1982: Open ocean modeling as an inverse problem: tidal theory. $J$. Phys. Oceanogr., 12, 1004-1018.

Blumberg, A. F., and G. L. Mellor, 1987: A description of a three-dimensional coastal ocean circulation model. In Three Dimensional Coastal Ocean Models, Vol. 4, Edited by N. Heaps, American Geophysical Union, Washington, DC, pp.208.

Bonekamp, H., G.J. van Oldenborgh and G. Burgers, 2001: Variational assimilation of TAO and XBT data in the HOPE OGCM, adjusting the surface fluxes in the tropical ocean. J. Geophys. Res., C106, 16693-16709.

Courtier, P., J.-N. Thépaut, and A. Hollingsworth, 1994: A strategy for operational implementation of 4DVar, using an incremental approach. Quart. J. Roy. Meteor. Soc., 120, 1367-1388

Das, S. K., and R. W. Lardner, 1991: On the estimation of parameters of hydraulic models by assimilation of periodic tidal data. . J. Geophys. Res., 96 (C8), 15187-15196.

Heemink, A. W., E. E. A. Mouthaan, M. R. T. Roest, E. A H. Vollebregt, K. B. Robaczewska, and $M$. Verlaam, 2002: Inverse 3D shallow water flow modeling of the continental shelf. Continental Shelf Research, 22, 465-484.

Holland, G. J., 1980: An Analytic model of the wind and pressure profiles in hurricanes. Mon. Wea. Rev., 108, 1212-1218.

Köhl, A., and D. Stammer, 2004: Optimal Observations for Variational Data Assimilation . J. Phys. Oceanogr., 34, 529-542.

LeDimet, F. X., and O. Talagrand, 1986: Variational algorithms for analysis and assimilation of meteorological observations: Theoretical aspects. Tellus, 38A, 97-110.

Lu, J., and W. E. Hsieh, 1997: Adjoint data assimilation in coupled atmosphere-ocean models:determining initial model parameters in a simple equatorial model. Quart. J. Roy. Meteor. Soc., 123, 21152139.

Lu, J., and W. E. Hsieh, 1998a: Adjoint data assimilation in coupled atmosphere-ocean models: determining initial conditions in a simple equatorial model. J. Met. Soc. Janpan, 76, 737-748.

Lu, J. and W. E. Hsieh, 1998b: On determining initial conditions and parameters in a simple couples atmospere-ocean model by adjoint data assimilation. Tellus, 50A, 534-544.

Martel, F., and C. Wunsch, 1993. The North Atlantic Circulation in the Early 1980s ? An Estimate from Inversion of a Finite-Difference Model. J. Phys. Oceanogr., 23, 898-924.

Mellor, G. L., and T. Yamada, 1982: Development of a turbulence closure model for geophysical fluid problems. Rev. Geophys. Space Phys., 20, 851875.

Mellor, G. L., 1989: Retrospect on ocenic boundary layer modeling an second moment closure. Hawaiian Winter Workshop on "Parameterization of small-scale processes", January 1989, University of Hawaii, Honolulu, Hawaii.

Mellor, G. L., 2003: Users guide for a threedimensional, primitive equation, numerical ocean model (June 2003 version), Prog. in Atmos. and Ocean. Sci, Princeton University, pp. 53.

Moore, A. M., H. G. Arango, E. Di Lorenzo, B. D. Cornuelle, A. J. Miller, and D. J. Neilson, 2004: A comprehensive ocean prediction and analysis system based on the tangent linear and adjoint of a regional ocean model. Ocean Modelling, 7, 227258.

Oldenborgh, G. Jan van, Gerrit Burgers, Stephan Venzke, Christian Eckert, and Ralf Giering, 1999: Tracking down the delayed ENSO oscillator with an adjoint OGCM. Mon. Wea. Rev., 127, 1477-1495.

Peng, M., L. Xie, and L.J. Pietrafesa, 2004: A numerical study of storm surge and inundation in the Croatan-Albemarle-Pamlico Estuary System. Estuarine, Coastal and Shelf Science, 59, 121-137.

Peng, M., L. Xie, and L. J. Pietrafesa, 2005: A Numerical Study on Hurricane Induced Storm Surge and Inundation in Charleston Harbor, South Carolina. J. Geophys. Res. (in review). 
Seiler, U., 1993: Estimation of open boundary conditions with the adjoint method. J. Geophys. Res., 98, 22855-22870.

Weaver, A. T., J. Vialard, and D. L. T. Anderson, 2003: Three- and four-dimensional variational assimilation with an ocean general circulation model of the tropical Pacific Ocean. Part 1: formulation, internal diagnostics and consistency checks. Mon. Wea. Rev ., 131, 1360-1378.

Xie, L., L. J. Pietrafesa, and M. Peng, 2004: Incorporation of a mass-conserving inundation scheme into a three-dimensional storm surge model. J. Coastal Research, 20, 1209-1223.

Yu, L., and J.J. O'Brien, 1991: Variational estimation of the wind stress drag coefficient and the oceanic eddy viscosity profile. J. Phys. Oceanogr., 21, 709-719.

Yu, L., and J.J. O'Brien, 1992: On the initial condition in parameter estimation. J. Phys. Oceanogr., 22, 1361-1364.

Zhang, A., B. B. Parker, and E. Wei, 2002: Assimilation of water level data into a coastal hydrodynamic model by an adjoint optimal technique. Continental Shelf Research, 22, 1909-1934.

Zhang, A., E. Wei, and B. B. Parker, 2003: Optimal estimation of tidal open boundary conditions using predicted tides and adjoint data assimilation technique. Continental Shelf Research, 23, 10551070. 